\title{
Timeline of the Works on the Correlation between COVID-19 and Y-DNA Haplogroup R1b
}

\author{
Sebastiano Schillaci* \\ February 16, 2021 (Version 1)
}

\begin{abstract}
Soon after the beginning of COVID-19 pandemic, many works have been reporting a yet-to-be-explained correlation of COVID-19 prevalence and severity with Y-DNA haplogroup R1b frequency. Here a chronologically ordered list of such works is provided.
\end{abstract}

\section{Introduction}

Because of the unusual spreading pattern of COVID-19 in Italy and in the world, at the beginning of April, I started wondering whether there was an underlying genetic reason for that. Watching genetic maps, I quickly noticed an apparent correlation with Y-DNA haplogroup R1b. On April 13th, 2020, I set up a website ${ }^{[4]}$ to publicize such discovery, joined a COVID-related Facebook group and posted my theory there ${ }^{[5]}$.

Initially, I shared my idea hoping someone would help me to develop it, but people seemed skeptical about it. After trying for a while to convince them, I decided to go on by myself. Using all the freely available data I could gather, I tried to verify the correlation statistically. As far as I know, my article ${ }^{[19]}$ has been the first serious attempt to mathematically 'prove' the existence of such a correlation. With further researches I later found out that other people had also noticed the same or similar correlations - most of them, I presume, independently from me.

*EMAIL ADDRESS: sebastiano_schillaci@yahoo.com
My initial plan was to assess the correlation between basic reproduction numbers $R_{0}$ and haplogroup percentages. Ideally, it would have been better to do it at a local level, so to compare data collected in a more uniform way. Unfortunately, there were not enough data for the haplogroup distributions inside countries or regions, but only for whole countries and populations (and only for the main subclades).

Besides, after searching for a while, I realized it was also difficult to find enough good quality estimates of basic reproduction numbers. So I resolved to use the initial growth rates of contagion (and deaths) for different countries, instead. To prevent possible criticisms, I calculated them using exactly the same approach already employed in another article assessing correlations with different factors; interestingly enough, the estimates chosen for the free parameters (days and cases) tend to be locally optimal for R1b correlations.

While writing my article I have started keeping track of related works, for easier reference. The following table contains an annotated list, in chronological order, of all the mentions to this theory I learned about. The milestones are highlighted and the specified date is the earliest I could find a reference for. 


\begin{tabular}{|c|c|c|c|}
\hline DATE & Author & TYPE & Notes \\
\hline 22 March 2020 & Maria del Pilar Corena-McLeod & Thread $^{[1]}$ & Incorrectly refers to the mitochondrial haplogroup $\mathrm{R} 1 \mathrm{~b}$ \\
\hline 30 March 2020 & Gigi Tevzadze & Article $^{[2]}$ & Marginal reference, mainly on haplogroup $\mathrm{G}$ \\
\hline 31 March 2020 & Paolo Sizzi & Thread $^{[3]}$ & Refers to Italy \\
\hline 13 April 2020 & Sebastiano Schillaci & Webpage $^{[4]}$ & Main points of the theory and visual comparison $(10+$ countries $)$ \\
\hline $\begin{array}{r}14 \text { April } 2020 \\
\text { (submitted on } 13 \text { April 2020) }\end{array}$ & Sebastiano Schillaci & Thread $^{[5]}$ & Shared webpage on Facebook Group physicists against sars-cov-2 \\
\hline 17 April 2020 & Sebastiano Schillaci & Thread $^{[6]}$ & $\begin{array}{l}\text { Consulted Mauricio Lucioni Maristany (Maulucioni), main con- } \\
\text { tributor to the world R1b map }{ }^{[7]}\end{array}$ \\
\hline 19 April 2020 & Ángel Gómez Moreno & Article ${ }^{[8]}$ & Refers to Europe and Africa with main focus on Spain $(1 / 3)$ \\
\hline 19 April 2020 & Alberto Pérez de Vargas & News ${ }^{[9]}$ & \\
\hline 24 April 2020 & Frédéric Boyer & Blog Post ${ }^{[10]}$ & Refers to Europe \\
\hline 25 April 2020 & Mohammed Hassai Awli & Article ${ }^{[11]}$ & Mostly diagrams \\
\hline 25 April 2020 & Miquel Hernandis & News $^{[12]}$ & \\
\hline 26 April 2020 & Johann Strauss & Blog Post ${ }^{[13]}$ & Relationship with BCG vaccine \\
\hline 27 April 2020 & Arsenio Escolar & News ${ }^{[14]}$ & \\
\hline 29 April 2020 & Anonymous (user4682) & Thread $^{[15]}$ & Graphical correlation with deaths per capita (20 countries) \\
\hline 5 May 2020 & Ángel Gómez Moreno & Article ${ }^{[16]}$ & Adds further European and African countries $(2 / 3)$ \\
\hline 19 May 2020 & Antonio Caselles Moncho & Article $^{[17]}$ & Multiple linear regression (?) (10 countries) \\
\hline 21 May 2020 & Frank Wallace Bentrem & Article ${ }^{[18]}$ & Graphical correlation with deaths per capita (57 countries) \\
\hline 22 May 2020 & Sebastiano Schillaci & Article ${ }^{[19]}$ & $\begin{array}{l}\text { Quantitative evaluation of the correlation in Italy and in the } \\
\text { world ( } 84 \text { countries) }\end{array}$ \\
\hline 23 May 2020 & Sebastiano Schillaci & Article ${ }^{[19]}$ & $\begin{array}{l}\text { Submitted to bioRxiv, but refused because it 'does not accept } \\
\text { preprints of articles reporting epidemiological studies' }\end{array}$ \\
\hline 25 May 2020 & Juan-Ramón Lacadena & Blog Post $[20]$ & Refers to previous works \\
\hline 26 May 2020 & Sebastiano Schillaci & Article ${ }^{[19]}$ & Submitted to Open Science Framework (OSF) \\
\hline 27 May 2020 & Sebastiano Schillaci & Thread $^{[21]}$ & Shared article on Facebook Group physicists against sars-cov-2 \\
\hline 5 June 2020 & Ángel Gómez Moreno & Article ${ }^{[22]}$ & Adds United States, Brazil and Japan (3/3) \\
\hline 8 June 2020 & John Allen & Article ${ }^{[23]}$ & Attempt at finding the involved genes \\
\hline $\begin{array}{r}19 \text { June } 2020 \\
\text { (submitted on } 10 \text { June } 2020 \text { ) }\end{array}$ & Mariya V. Ragulskaya & Article $^{[24]}$ & Refers to haplogroup R1a and R1b (peer-reviewed) \\
\hline 27 June 2020 & Sebastiano Schillaci & Article $^{[19]}$ & 2nd version \\
\hline $\begin{array}{r}17 \text { July } 2020 \\
\text { (submitted on } 20 \text { June } 2020 \text { ) }\end{array}$ & O. Abu Hammad et al. & Article ${ }^{[25]}$ & Marginal reference to previous works (peer-reviewed) \\
\hline 20 July 2020 & Spyros Anagnostou & Article ${ }^{[26]}$ & Graphical correlation with deaths per capita (38 countries) \\
\hline 20 July 2020 & Ángel Gómez Moreno & Article $^{[27]}$ & Compares Western European and non-European countries \\
\hline 29 July 2020 & Sebastiano Schillaci & Article ${ }^{[19]}$ & 3rd version \\
\hline 30 July 2020 & Sebastiano Schillaci & Thread $^{[28]}$ & Shared article on Facebook Group Statistiche Coronavirus Italia \\
\hline 10 August 2020 & Dave Dalton & Article $^{[29]}$ & Marginal reference, mainly on haplogroup R1a \\
\hline $\begin{array}{r}21 \text { August } 2020 \\
\text { (submitted on } 27 \text { May } 2020 \text { ) }\end{array}$ & J.R. Delanghe et al. & Article $^{[30]}$ & $\begin{array}{l}\text { Quantitative evaluation of the correlation in the Netherlands, } \\
\text { Belgium and in the world ( } 28 \text { countries) (peer-reviewed) }\end{array}$ \\
\hline 13 October 2020 & G. Fazio et al. & Article $^{[31]}$ & Refers to Italy \\
\hline $\begin{array}{r}15 \text { October } 2020 \\
\text { (submitted on } 28 \text { June } 2020)\end{array}$ & L. Janda et al. & Article ${ }^{[32]}$ & Marginal reference to previous works (peer-reviewed) \\
\hline 20 October 2020 & Aleš Žužek & News ${ }^{[33]}$ & \\
\hline 23 October 2020 & M. Montopoli et al. & Article ${ }^{[34]}$ & Response to J.R. Delanghe et al.'s article (peer-reviewed) \\
\hline 26 October 2020 & Nacho de Blas & Blog Post ${ }^{[35]}$ & Marginal reference to previous works \\
\hline 9 November 2020 & Sandro Modeo & News ${ }^{[36]}$ & \\
\hline 12 November 2020 & Nacho de Blas & TV News ${ }^{[37]}$ & \\
\hline 13 November 2020 & Ole Bernt Lenning & Seminar ${ }^{[38]}$ & Refers to the world \\
\hline 14 November 2020 & Luis Ordóñez & News ${ }^{[39]}$ & \\
\hline 23 November 2020 & Nacho de Blas & Blog Post ${ }^{[40]}$ & Refers to previous works \\
\hline 10 December 2020 & Vasilis S. Gavalas & Article ${ }^{[41]}$ & Assessment of NPIs efficacy on the basis of previous works \\
\hline 23 December 2020 & Sandro Modeo & News ${ }^{[42]}$ & \\
\hline 27 December 2020 & José Manuel Etxaniz Makazaga & News ${ }^{[43]}$ & \\
\hline 8 January 2021 & Peter Mühlbauer & News ${ }^{[44]}$ & \\
\hline 14 January 2021 & Peter Mühlbauer & News $^{[45]}$ & \\
\hline 3 February 2021 & Sandro Modeo & News ${ }^{[46]}$ & \\
\hline
\end{tabular}

Table: Timeline of the works on COVID-19 and Y-DNA haplogroup R1b. 


\section{References}

[1] Corena-McLeod, M. del P. Wondering if anyone is doing research on mitochondrial haplogroup $R 1 b$ and its relationship with COVID-19 incidence and symptom severity?. ResearchGate. March 22, 2020. https:// www.researchgate.net/post/Wondering if anyone $i$ $s$ doing research on mitochondrial haplogroup $\bar{R}$ $1 \bar{b}$ _and_its_relationship_with_COVID-19_incidence _and_symptom_severity

[2] Tevzadze, G. Haplogeography of COVID-19: A hypothesis. ResearchGate. March 30, 2020. https://doi.org/ 10.13140/RG.2.2.17496.44803

[3] Sizzi, P. Tweet (in Italian). Twitter. March 31, 2020. https://twitter.com/granlombard/status/12450857965 43205376

[4] Schillaci, S. Possible link between COVID-19 susceptibility and genetic factors. SXS. April 13, 2020. http://sxs.altervista.org/coronavirus/

[5] Schillaci, S. physicists against sars-cov-2. Facebook Groups. April 14, 2020. https://www.facebook.com/g roups/PhysicistsAgainstSARSCoV2/permalink/839378 729887388

[6] Schillaci, S. User talk:Maulucioni. Wikimedia Commons. April 17, 2020. https://commons.wikimedia.or g/wiki/User_talk:Maulucioni\#R1b_distribution

[7] Wikimedia Commons contributors. Haplogroup R1b ( $Y$ $D N A)$. Wikimedia Commons. July 28, 2019. https: //upload.wikimedia.org/wikipedia/commons/e/ec/Hapl ogroup_R1b_(Y-DNA).PNG

[8] Gómez Moreno, Á. Coronavirus, Population Genetics, and Humanities. Mirabilia Journal. Eletronic Journal of Antiquity \& Middle Ages, 30. April 22, 2020. https: //www.revistamirabilia.com/sites/default/files/pdfs/01 -_gomezmoreno.pdf

[9] Pérez de Vargas, A. Este virus tiene sus preferencias (in Spanish). Europa Sur. April 19, 2020. https://ww w.europasur.es/opinion/articulos/virus-preferencias-ca mpo-chico_0_1456654673.html

[10] Boyer, F. Vulnérabilité au Coronavirus : une question de génétique? (in French). AgoraVox. April 24, 2020. https://www.agoravox.fr/tribune-libre/article/vulnerabil ite-au-coronavirus-une- 223624

[11] Hassai Awli, M. Examining the statistical correlation between Haplogroup $Y$ DNA, R1b and infection by covid 19 virus. LinkedIn. April 25, 2020. https://www.link edin.com/posts/mohammedhassai123_our-genes-and-c ovid19-activity-6750473781648596992-5HYe

[12] Hernandis, M. De la peste negra a una pista genética para el Covid-19 (in Spanish). El Mundo. April 25, 2020. https://www.elmundo.es/comunidad-valenciana/ alicante/2020/04/25/5ea330e321efa0435e8b4645.html
[13] Strauss, J. Covid Infection Scenarios. LinkedIn. April 26, 2020. https://www.linkedin.com/pulse/covid-infecti on-scenarios-johann-strauss $/$ ? articleld $=6660159729681$ 215489

[14] Escolar, A. Europa Occidental, más proclive al coronavirus por genética (in Spanish). elDiario.es. April 27, 2020. https://www.eldiario.es/arsenioescolar/europa-oc cidental-proclive-coronavirus-genetica_132_5919073.h tml

[15] user4682. Coronavirus deaths correlated to population wide genetic markers. Hacker News. April 29, 2020. https://imgur.com/a/vrgEa8I

[16] Gómez Moreno, Á. Coronavirus and Genetics: in no way a miracle. Mirabilia Journal. Eletronic Journal of Antiquity \& Middle Ages, 30. May 5, 2020. https: //www.revistamirabilia.com/sites/default/files/pdfs/02 _gomezmoreno.pdf

[17] Caselles Moncho, A. Un statistical explanation. Avances Sistémicos, 8, 6-7. May 19, 2020. https://www.sesge. org/images/docs/AvancesSistemicos/avances_sistemico s_8.pdf

[18] Bentrem, F. W. COVID-19 Death Rate: Is it in our $D N A$ ? . ResearchGate. May 31, 2020. https://doi.org/ 10.13140/RG.2.2.29960.65289/1

[19] Schillaci, S. Possible Correlation between COVID-19 Contagion and Y-DNA Haplogroup R1b. OSF Preprints. May 26, 2020. https://doi.org/10.31219/osf.io/yv8kc

[20] Lacadena, J.-R. Coronavirus y divagaciones genéticas (in Spanish). Real Academia Nacional de Farmacia. May 25, 2020. https://www.ranf.com/noticia/coronav irus-genetica/

[21] Schillaci, S. physicists against sars-cov-2. Facebook Groups. May 27, 2020. https://www.facebook.com/g roups/PhysicistsAgainstSARSCoV2/permalink/869162 470242347

[22] Gómez Moreno, Á. COVID-19 and Population Genetics: Correlation, Causation and Likelihood (Third and last delivery). Mirabilia Journal. Eletronic Journal of Antiquity \& Middle Ages, 30, 316-343. June 5, 2020. https://www.revistamirabilia.com/sites/default/files/p dfs/15._covid6669.pdf

[23] Allen, J. COVID-19 Infection via a Gene Variant. OSF Preprints. June 8, 2020. https://doi.org/10.31219/osf .io/njsk8

[24] Ragulskaya, M. V. Space weather and COVID-19 Pandemic Genogeography. Journal of Novel Physiotherapy and Physical Rehabilitation, 7(1), 031-032. June 19, 2020. https://doi.org/10.17352/2455-5487.000074

[25] Abu Hammad, O., Alnazzawi, A., Borzangy, S. S., et al. Factors Influencing Global Variations in COVID-19 Cases and Fatalities; A Review. Healthcare, 8(3), 216. July 17, 2020. https://doi.org/10.3390/healthcare80 30216 
[26] Anagnostou, S. The Unexplained East-West Dichotomy in COVID-19 Incidence in Europe: Exploring the R1b $Y$-DNA Haplogroup Hypothesis. Journal of Regional Socio-Economic Issues, 10(2), 40-47. July 20, 2020. https://geography.aegean.gr/files/labs/Journal__Vol ume--X__ssue--2__June__ $2020_{-}$Online.pdf

[27] Gómez Moreno, Á. España y la Gran Crisis (2020) (in Spanish). Tiempo de Paz, 137, 107-115. July 20, 2020. https://fundadeps.org/wp-content/uploads/2020/07/T P-137-PDF.pdf

[28] Schillaci, S. Statistiche Coronavirus Italia (in Italian). Facebook Groups. July 30, 2020. https://www.facebo ok.com/groups/StatisticheCoronavirusltalia/permalink /617581299173806

[29] Dalton, D. Why People of Bangladeshi Origin Are Most at Risk of Dying from COVID-19 in the UK, i.e., Why They Carry the Highest Level of Those Six Deleterious Neanderthal-Inherited Genes. SSRN. August 10, 2020. https://doi.org/10.2139/ssrn.3673483

[30] Delanghe, J. R., Buyzere, M. L. D., Bruyne, S. D., et al. The potential influence of human $Y$-chromosome haplogroup on COVID-19 prevalence and mortality. Annals of Oncology, 0(0). August 21, 2020. https: //doi.org/10.1016/j.annonc.2020.08.2096

[31] Fazio, G., and Poidomani, G. Inter-regional italian differences about COVID-19 disease. SISMED - Società Italiana Scienze Mediche. October 13, 2020. https: //www.sismed-it.com/inter-regional-italian-differences -about-covid-19-disease/

[32] Janda, L., Mihalcin, M., and Štastná, M. Is a healthy microbiome responsible for lower mortality in COVID19?. Biologia, 76(2), 819-829. October 15, 2020. ht tps://doi.org/10.2478/s11756-020-00614-8

[33] Žužek, A. Iskanje rešilne bilke, ki bo unicila korono (in Slovenian). Siol.net. October 20, 2020. https: //siol.net/novice/svet/iskanje-resilne-bilke-ki-bo-unicila -korono-536997

[34] Montopoli, M., Zumerle, S., Rugge, M., et al. Genetic and hormonal influence on SARS-CoV-2-infection susceptibility. Annals of Oncology, 31(11), 1584-1585. November 1, 2020. https://doi.org/10.1016/j.annonc .2020.07.022

[35] de Blas, N. Reflexiones sobre el COVID-19 de un epidemiólogo veterinario (in Spanish). Working in Epidemiology. October 26, 2020. http://www.winepi.net /covid19.htm

[36] Modeo, S. Cosi ci ha colpito la «seconda ondata» (e cosa può succedere ora) (in Italian). Corriere della Sera. November 9, 2020. https://www.corriere.it/salute/m alattie infettive/20_novembre_09/covid-cosi-ci-ha-tra volti-seconda-ondata-che-cosa-puo-succedere-ora-654d 88ba-202f-11eb-a173-71e667bc7224.shtml
[37] de Blas, N. "Hay un grupo genético más predispuesto a pasar la COVID-19, frecuente en País Vasco" (in Spanish). EITB Radio Televisión Pública Vasca. November 12, 2020. https://www.eitb.eus/es/television/program as/en-jake/videos/detalle/7629313/video-nacho-blas-e pidemiologo-relacion-genetica-incidencia/

[38] Lenning, O. B. CeFH Genetics Friday: A possible covariation between COVID19 lethality and Y Chromosome R1b in perspective of the epidemiological situation. Norwegian Institute of Public Health. November 13, 2020. https://www.fhi.no/en/more/research-centres/Ce ntre-for-fertility-and-health/events-en/cefh-genetics-fr iday-a-possible-covariation-between-covid19-lethality-an $\mathrm{d}-\mathrm{y} /$

[39] Ordóñez, L. «Apenas se está considerando que podría haber una predisposición genética al contagio» (in Spanish). La Voz de Asturias. November 14, 2020. https://www.lavozdeasturias.es/noticia/asturias/2020/1 $1 / 12$ /apenas-considerando-haber-predisposicion-geneti ca-contagio/00031605203645867758375.htm

[40] de Blas, N. Reflexiones sobre el COVID-19 de un epidemiólogo veterinario (in Spanish). Working in Epidemiology. November 23, 2020. http://www.winepi .net/covid19.htm

[41] Gavalas, V. S. How effective are the lockdown and quarantine of the general population during the COVID19 pandemic?. ResearchGate. December 10, 2020. https://doi.org/10.13140/RG.2.2.22768.69123

[42] Modeo, S. Quando finirà il Covid? Le tappe (e gli ostacoli) per uscire dall'incubo (in Italian). Corriere della Sera. December 23, 2020. https://www.corrie re.it/salute/20_dicembre_23/covid-quando-finira-tapp e-ostacoli-uscire-dall-incubo-219ad926-42e7-11eb-a38878033ff67873.shtml

[43] Etxaniz Makazaga, J. M. De prisas, genética y vacunas (in Spanish). Noticias de Gipuzkoa. December 27, 2020. https://www.noticiasdegipuzkoa.eus/actualidad/ sociedad/2020/12/27/prisas-genetica-vacunas/1078068. html

[44] Mühlbauer, P. Die schlimmste Seuchenkatastrophe der Geschichte (in German). Telepolis. January 8, 2021. https://www.heise.de/tp/features/Die-schlimmste-Seuc henkatastrophe-der-Geschichte-5014534.html

[45] Mühlbauer, P. Irland und Corona: falsche Weihnachtsunterbrechung oder falsche Maßnahme? (in German). Telepolis. January 14, 2021. https://www.heise.de/t $\mathrm{p} /$ features/Irland-und-Corona-falsche-Weihnachtsunter brechung-oder-falsche-Massnahme-5024336.html

[46] Modeo, S. Cosi le varianti del coronavirus tentano di aggirare $i$ vaccini (in Italian). Corriere della Sera. February 3, 2021. https://www.corriere.it/salute/malattie_i nfettive/21 febbraio 03/cosi-coronavirus-tenta-ogni-is tante-aggirare-vaccini-246bb88c-64a7-11eb-aad7-ece68 84524fa.shtml 